vol. $27-n^{\circ} 2$ | 2011

Numéro ouvert

\title{
Satisfacción residencial de los africanos en Andalucía (España)
}

La satisfaction résidentielle des Africains en Andalousie (Espagne)

Residential Satisfaction of Africans in Andalusia (Spain)

Juan Carlos Checa Olmos, Ángeles Arjona Garrido y Francisco Checa y Olmos

\section{OpenEdition}

\section{Edición electrónica}

URL: https://journals.openedition.org/remi/5465

DOI: $10.4000 /$ remi. 5465

ISSN: $1777-5418$

Editor

Université de Poitiers

Edición impresa

Fecha de publicación: 1 octubre 2011

Paginación: 161-182

ISBN: 979-10-90426-01-6

ISSN: 0765-0752

\section{Referencia electrónica}




\title{
Note de recherche
}

\section{Satisfacción residencial de los africanos en Andalucía (España)}

\author{
Juan Carlos CHECA OLMOS ${ }^{1}$, Ángeles ARJONA \\ GARRIDO $^{2}$ y Francisco CHECA y OLMOS $^{3}$
}

\section{INTRODUCCIÓN}

ndalucía ha sido, tradicionalmente, expulsora de población en migraciones que han tenido como destino tanto el exterior: América Central y Sur, Europa y norte de África, como el interior: Cataluña, Madrid y País Vasco. Pero en las dos últimas décadas se ha convertido en una de las comunidades autónomas que mayor número de extranjeros recibe en España ${ }^{4}$, bien en calidad de turistas en busca de sol y playa, bien de asalariados que se emplean, principalmente, en la agricultura, construcción o sector servicios.

Además de la diversidad de lugares de origen, la inmigración en Andalucía se caracteriza, entre otros aspectos, por su distribución geográfica, que responde a las características y demandas de los mercados de trabajo anteriormente señalados. Esto es, el stock de población se concentra fundamentalmente en los municipios y ciudades de la costa, así como en las capitales de provincia y lugares de agricultura intensiva o extensiva con demanda de mano de obra (Mapas 1 y 2).

1 Profesor titular Sociología, Universidad de Almería, Edificio C Humnidaddes II, La Cañada de San Urbano S/N, 04120 Almería, Spain; jcheca@ual.es

2 Profesora titular Antropología Social, Universidad de Almería, Edificio C Humnidaddes II, La Cañada de San Urbano S/N, 04120 Almería, Spain; arjona@ual.es

3 Profesor titular Antropología Social, Universidad de Almería, Edificio C Humnidaddes II, La Cañada de San Urbano S/N, 04120 Almería, Spain; fcheca@ual.es

4 Según el Instituto Nacional de Estadística, a principios de 2009 los extranjeros en Andalucía ascendían a 668 093, lo que equivale al 13,3\% de la población. Estas cifras sólo son superadas por Cataluña, Madrid, y Comunidad Valenciana con 1184 192, 1043133 y 882870 extranjeros, respectivamente. 
Ahora bien, la incorporación residencial de los inmigrados - especialmente la procedente de África - no ha experimentado un proceso de inserción satisfactorio, tanto por sus niveles de segregación, como por la calidad de las viviendas ocupadas (Checa y Olmos, Checa y Arjona, 2009). Los africanos se han ubicado tanto en los diseminados, que se esconden en el laberinto de caminos y veredas que dibujan las explotaciones agrícolas, como en las zonas más deterioradas de los núcleos urbanos. De manera que se rompe, en muchos casos, con el principio que define y entiende la vivienda como una necesidad primaria donde se proporciona el aislamiento y privacidad necesaria para el desarrollo personal - vivienda digna - y, por otro, una serie de necesidades subjetivas de orden secundario - o primario - a través de las cuales el individuo ve realizadas sus aspiraciones y cumplidos sus deseos.

\section{Mapa 1: Número de extranjeros en Andalucía por municipios en 2007}

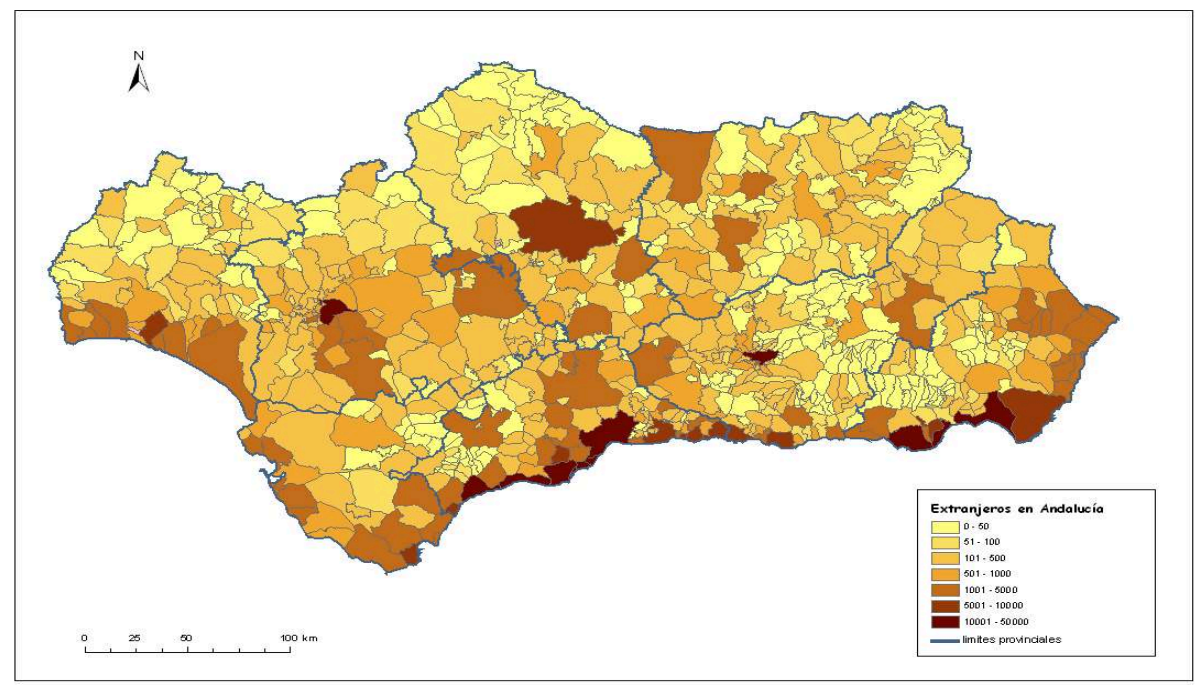

Fuente: Padrón municipal; elaboración propia 


\section{Mapa 2: Porcentaje de extranjeros en Andalucía por municipios en 2007}

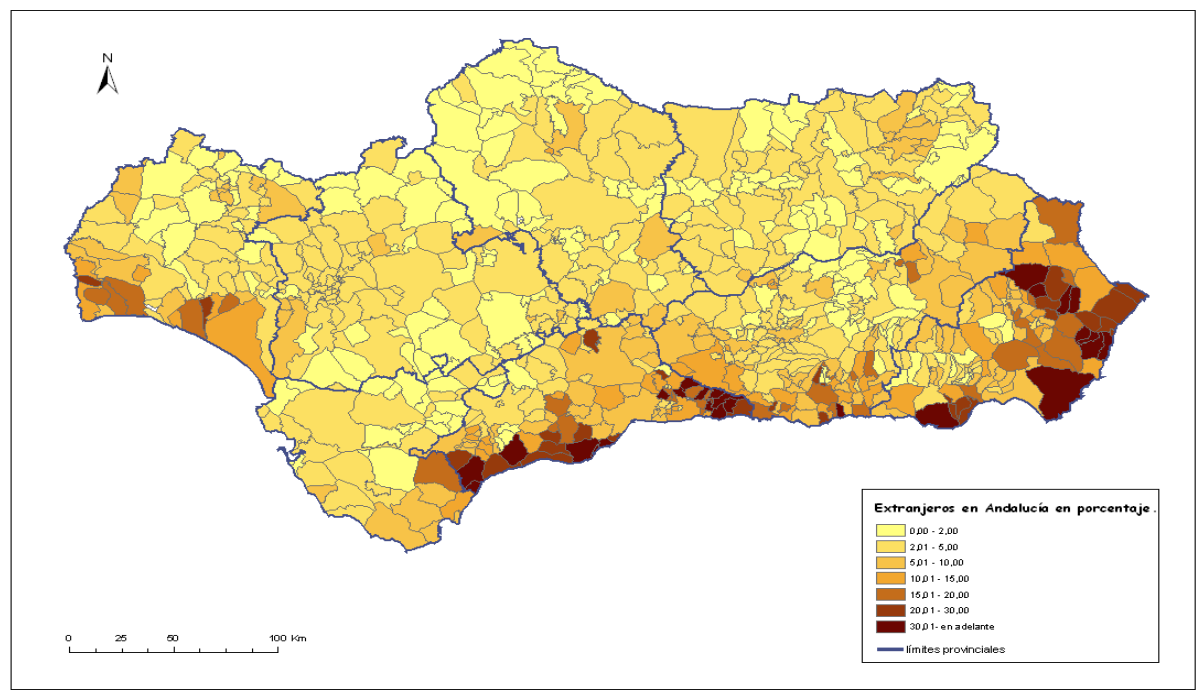

Fuente: Padrón municipal; elaboración propia

Entendemos, por tanto, como vivienda digna aquella que posibilita el desarrollo normal de sus habitantes o moradores, a partir del cumplimiento de los siguientes requisitos básicos: estabilidad, accesibilidad, habitabilidad y adecuación (Kenna, 2006). Reconocemos, pues, a la vivienda como un derecho humano ${ }^{5}$, que supone, por tanto, romper con cualquier posible neutralidad. Más aún, concebimos por dicho derecho como el remedio(s) - arquitectura de remedios - a tomar para solventar una situación de injusticia social.

En el marco y definición de la vivienda como adecuada, las soluciones a sus deficiencias, además de los materiales de construcción y las políticas en que se apoyan, debe aparecer tanto la expresión de identidad que se deriva de su ocupación, como la diversidad cultural. Por tanto, en la esfera residencial se debe velar para que no se sacrifiquen las dimensiones culturales de la vivienda, y es aquí donde toma sentido la percepción subjetiva del espacio por parte del morador; más concretamente, la satisfacción residencial es un elemento fundamental a la hora de dignificar una vivienda.

$5 \mathrm{Al}$ menos el $40 \%$ de las Constituciones del mundo contienen referencias a la vivienda o a los derechos a la vivienda (Leckie, 2000). En la Constitución Española en el título primero «De los derechos y deberes fundamentales», en su artículo 47 se afirma que «Todos los españoles tienen derecho a una vivienda digna y adecuada». Ahora bien, ¿significa esto que los no españoles extranjeros - no tienen derecho a la vivienda digna? La ley orgánica 8/2000 sobre derechos y libertades de los extranjeros en España de 22 de diciembre, sostiene que los extranjeros «residentes» tienen «derecho a acceder al sistema público de ayudas en materia de vivienda en las mismas condiciones que los españoles». 
A pesar de la procedencia diversa de inmigrantes en Andalucía en este texto vamos a analizar sólo la satisfacción residencial que tienen los africanos asentados en Andalucía ${ }^{6}$. No nos detenemos en el resto de población extranjera puesto que su situación residencial es sustancialmente mejor (Checa Olmos, Checa y Arjona, 2009). Apenas hay europeos del Este o Latinoamericanos viviendo en diseminados. Además, sólo en ciertos barrios de los núcleos urbanos los africanos comparten espacio con otros inmigrantes. E incluso, cuando esto último ocurre, no podemos hablar que las características de habitabilidad de las viviendas sean iguales. Situación que se explica por la capacidad de endeudamiento de unos colectivos frente a otros y por la acogida del contexto de recepción. De manera que los mercados laborales donde se emplean los europeos y latinos tienen mayores recompensas económicas; y los autóctonos los prefieren como vecinos frente a los africanos.

En definitiva, con este trabajo se pretende comprobar cómo el espacio determina o puede determinar - la exclusión o anexión de los africanos a su entorno. E intentamos cubrir un vacío empírico, especialmente en la literatura española, sobre la satisfacción residencial puesto que, aunque no han faltado investigaciones que pongan el acento en las condiciones objetivas de habitabilidad (Colectivo Ioé, 2005; Rinken y Herrón, 2004; Checa y Arjona, 2005), ninguna ha hecho referencia a la satisfacción residencial en un sentido amplio ${ }^{7}$.

\section{ESTRUCTURA TEÓRICA}

El estudio de la satisfacción residencial combina dos conceptos - satisfacción y residencia - que, por si solos, han generado un desarrollo teórico y conceptual propio. Aquí, por razones obvias, sólo vamos a desbrozar sus elementos clave, para centrarnos más en los resultados de su combinación.

La satisfacción es uno de los indicadores subjetivos más importantes para medir la calidad de vida de los sujetos. Por tanto, esta temática cuenta con un ingente corpus bibliográfico que aborda su definición. De ahí que podamos encontrar dos grandes corrientes a la hora de afrontarla - medirla - y entenderla. Por un lado, la perspectiva cognitiva ha entendido la satisfacción atendiendo tanto al nivel de aspiración como al de privación. Así, la satisfacción puede ser definida como la discrepancia percibida entre lo aspirado y el logro (Campbell, Converse y Rodgers, 1976). Entre las esperanzas individuales de acceder a algo y la percepción de lo conseguido, con lo cual la satisfacción implica una experiencia de juicio. Por otro, y relacionado con lo anterior, la privación de lo aspirado es un indicador claro de la aparición de sentimientos; esto es, tiene que ver más con el afecto que con lo cognitivo (Weidemann y Anderson, 1985). Más concretamente, la satisfacción es un estado positivo y, por tanto, afectivo, con respecto a lo aspirado.

6 Los resultados corresponden a la investigación Vivienda, discriminación y segregación espacial de los inmigrados en Andalucía, financiado por la Subdirección General de Proyectos de Investigación Científica y Técnica del Ministerio de Educación y Ciencia, dentro del programa nacional de Estudios Sociales y Económicos (I+D).

7 Hasta el momento sólo Checa (2007) y Checa y Arjona (2005) han estudiado la satisfacción residencial de los africanos en la provincia de Almería, haciendo referencia, exclusivamente, a la vivienda y como respuesta a una escala directa. 
En consecuencia, lo que parece claro es que ambas perspectivas son confluyentes, ya que la satisfacción proviene de procesos - fruto de una evaluación - pero el estado es totalmente afectivo - sentimiento positivo/negativo.

En cuanto al segundo concepto, la vivienda es más que una estructura física, puesto que se ha convertido en una mercancía que actúa como medio para ganar dinero, a la vez que se comporta como indicador socioeconómico; es decir, la vivienda presenta una relación con la estructura social donde se enmarca. Además, la concepción tradicional del problema de la vivienda ha sido definido básicamente como un desequilibrio entre lo necesitado y lo producido, sin tener en cuenta la relación o integración entre la vivienda y la estructura social (Kemeny, 1992). De ahí que el concepto de residencia - o de habitar (Cortés, 1995) - se establece como elemento aglutinador entre las dimensiones sociales y físico-espaciales.

En resumen, ambos elementos - satisfacción y residencia - se combinaron cuando fue preciso dar respuesta a la necesidad de encontrar criterios adecuados que evalúen las condiciones de vivienda para mejorar la calidad de vida de los moradores ${ }^{8}$. Si inicialmente el entorno residencial sólo se catalogaba a partir de criterios objetivos: año de construcción, estilo arquitectónico, estructura espacial, espacios verdes o localización geográfica, en la actualidad está consensuado que en la satisfacción residencial actúan otras variables referidas al usuario. Éstas pueden tener un carácter tanto objetivo como subjetivo; por ello, actúan variables como ingresos, edad, sexo, composición poblacional del vecindario y, por supuesto, la valoración que los usuarios hacen de las características físicas y sociales del entorno. Hasta el punto que cabe la posibilidad de que en un vecindario con composición homogénea sus vecinos manifiesten valoraciones diferentes en base a la rutina, aspiraciones, etc. (Karsten et al., 2006).

El interés científico por la satisfacción residencial arranca a partir de la década de los sesenta ${ }^{9}$, si bien es cierto que con el paso del tiempo la literatura se ha ido reduciendo hasta el punto que apenas se encuentran investigaciones en la última década ${ }^{10}$. En cualquier caso éstas y otras se pueden agrupar, tal y como sostienen Weidemann y Anderson (1985), en dos categorías: una, aquellas investigaciones que evalúan criterios de cualidad residencial (por ejemplo, Marans y Rodgers, 1975; Galster y Hesser, 1981; Cutter, 1982, Gifford, 1987, Bonaiuto et al., 1999; Parkes et al., 2002; Pinquart y Burmedi, 2004), donde la satisfacción es la variable criterio y actúa como dependiente. Más concretamente, los investigadores establecen que los factores ambientales que determinan el grado o niveles de satisfacción con el entorno están definidos por variables como características físicas de la casa, composición poblacional del vecindario, estatus socioeconómico, etc.

8 Yancey (1971) muestra como en la concepción de la planificación urbana existen diferentes visiones entre los sujetos, que se derivan del estatus, sobre todo, entre los que planificaban (urbanistas y arquitectos) y los usuarios a quienes iban destinados, puesto que lo diseñado por los «expertos» no siempre creaba el nivel de satisfacción esperado, especialmente, cuando se construye para clases desfavorecidas.

9 Véanse, entre otros, Willmott y Young (1960), Fried y Gleicher (1961), Ryan (1963), Hollinshead y Rogler (1963).

10 Pueden verse, entre otros, Martinez, Felka y Simpson (1991); Bruin y Cook (1997), Carvalho, George y Anthony (1997), Rojo et al. (2001), Oh (2003), Phillips, Siu, Yeh y Cheng (2004), McCrea y Stimson (2005), Chapman y Lombard (2006), Adriaanse (2007), Baker (2008). 
$\mathrm{Y}$ dos, las que toman la satisfacción residencial como predictor de comportamiento, especialmente en forma de movilidad residencial (Speare, 1974; Newman y Duncan, 1979; Oh, 2003), por tanto, la satisfacción se comporta como definidor de conducta y opera como variable independiente. Específicamente, el ambiente actúa como elemento estresante, obligando al individuo a tomar una conducta de ajuste ante esa situación. Por ello, cuando un individuo tiene actitudes negativas hacia su entorno por el incumplimiento de necesidades, aspiraciones, expectativas, etc., trata de reducirlo a un estado temporal, cuya solución final es modificar las aspiraciones, adecuándolas a las posibilidades del ambiente o el traslado a otro ambiente.

Speare (1974) propone un modelo comportamental entre la satisfacción y el movimiento (Figura 1). Dicho modelo depende, primero, de factores individuales y/o familiares: idioma practicado, régimen de propiedad, estado civil, género, lugar de procedencia, ingresos, años de escolaridad, etc. De manera que hablar un idioma diferente en el barrio al practicado en casa, estar soltero, ser mujer, tener ingresos bajos y una tasa baja de escolaridad se convierten en variables que determinan, en mayor grado, el estrés residencial, que favorece el cambio de los sujetos a otro entorno/barrio que responda a sus necesidades.

Segundo, características de la ubicación: tipo de entorno, criminalización, desorden social y físico, vecindario y región. De ahí que en un medio aislado, con altas tasas de delitos y con un marco ambiental deteriorado la población presenta altas dosis de deseo de cambio.

Y tercero, los vínculos sociales que mantenga con su ambiente residencial, como son las relaciones de amistad, cohesión social, control social o actividad vecinal. Hasta el punto que cuanto los sujetos tienen un número considerable de amigos, que configuran una densa cohesión social con el entorno y con una alta participación asociativa la movilidad será más reducida.

Figura 1: Modelo toma de decisión de traslado

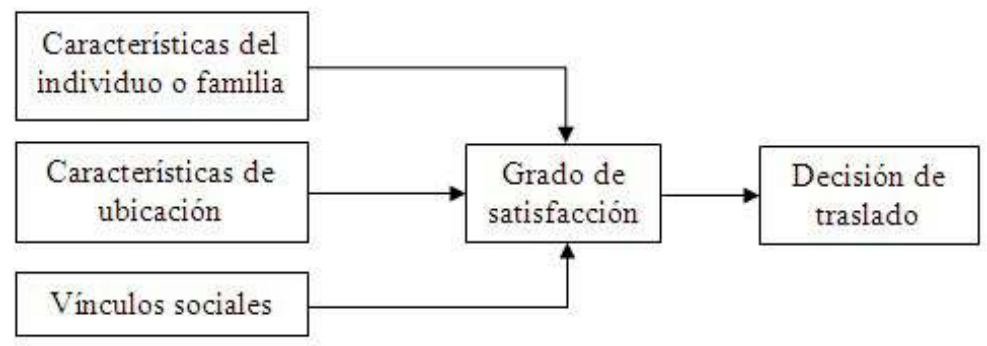

Fuente: Speare (1974: 176)

En definitiva, la movilidad se considera la única alternativa a la falta de ajuste entre los anteriores elementos y su entorno residencial. Por tanto, asume la incongruencia 
entre las necesidades y aspiraciones de los usuarios. Ahora bien, si la satisfacción residencial es, posiblemente, el mejor predictor a la hora de explicar el cambio de residencia migración local -, no lo es tanto en la decisión de migrar hacia otro país. Las migraciones internacionales, como refleja el vasto volumen de publicaciones, se deben, sobre todo, a cuestiones socioeconómicas, bien por aspiración a encontrar un mejor empleo o por encontrar alguna alternativa laboral. Por ello, con nuestro trabajo de campo, en ningún caso, encontramos a un africano que señalara la insatisfacción residencial con su vivienda de origen, como el elemento desencadenante de su migración.

En cualquier caso, la mayoría de la literatura sobre satisfacción residencial toma como referencia las características de los usuarios (cognitivo y/o comportamental) o bien el entorno (físico y social). Pero muy pocas son las investigaciones que han organizado esas variables en un modelo que relacione ambos elementos (Parkes et al., 2002). Van a ser los estudios de Amérigo (1990) y Amérigo y Aragonés (1997) los que proponen analizar dicha satisfacción a partir de la interacción de los diferentes procesos cognitivos, afectivos y comportamentales con el entorno más cercano (Figura 2).

De manera que los atributos objetivos del entorno, una vez que han sido evaluados - de manera subjetiva -, producen un determinado sentimiento de satisfacción, teniendo en cuenta que éstos siempre dependen de las características personales - especialmente sociodemográficas - de quienes emiten el juicio. El resultado de esa evaluación - positiva o negativa -, atendiendo a la experiencia individual, va a favorecer la interacción con su entorno o, por el contrario, se producirá un aliciente para la movilidad residencial en busca de una mayor satisfacción con la vida.

Figura 2: Modelo de satisfacción residencial

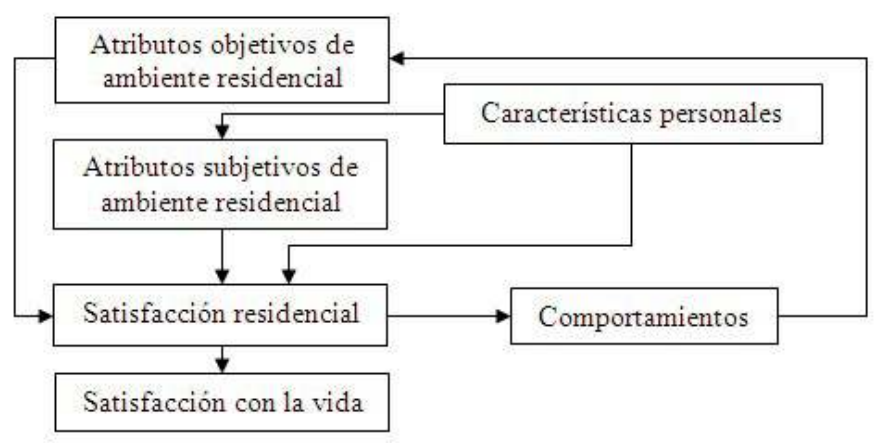

Fuente: Amérigo y Aragonés (1997)

Esta propuesta teórica - y metodológica - va a guiar nuestra investigación. Por ello, primero realizaremos una descripción objetiva de los entornos donde se asientan los africanos y, segundo, comprobaremos el grado de satisfacción. De manera que los resultados podrían servir de guía a la hora de abordar actuaciones futuras tanto a políticos, 
urbanistas o incluso arquitectos. A sabiendas que en los últimos años se están diseñando políticas de mixticidad urbanística (social mix) para potenciar la inclusión residencial de los inmigrantes, pero en ningún caso se tiene en cuenta la opinión - satisfacción - de los propios afectados. En consecuencia, hasta qué punto las políticas urbanísticas de mixticidad favorecen la integración residencial y las relaciones interétnicas sin contar con la propia definición de los afectados (Berube, 2005; Tunsall y Fenton, 2006).

\section{MÉTODO Y DATOS}

Para comprobar la satisfacción residencial de los africanos asentados en Andalucía hemos utilizado como técnica la encuesta. En la selección de los lugares donde se realiza la investigación tuvimos en cuenta varios criterios: en primer lugar, y a partir del Padrón de Habitantes, por un lado, el número de africanos y, por otro, la proporción que representan en los municipios. En segundo lugar, las ciudades y/o municipios que en el Decreto 2/2001 son considerados de alta movilidad laboral. Y, en tercer lugar, todas las capitales de provincia. De manera que la investigación se llevó a cabo en 47 municipios y/o ciudades de Andalucía ${ }^{11}$. La muestra se extrae a partir del número africanos, de cada uno de los municipios elegidos, en el año 2007, según el Padrón. El muestreo lo realizamos por cuotas con afijación proporcional, atendiendo al lugar de asentamiento y ubicación de las viviendas - diseminado o núcleo urbano - y origen - magrebíes y África subsahariana. Aunque, como veremos en el apartado de resultados, el origen de la población inmigrada es la única variable que no es significativa a la hora de predecir la satisfacción residencial.

Los puntos de muestreo en los núcleos urbanos se realizaron en las secciones con mayor densidad y concentración de africanos, desde donde se establecieron rutas aleatorias hasta cubrir las cuotas pertinentes. Para los diseminados nos valimos de fotos aéreas, a la vez que dividimos los términos municipales en kilómetros cuadrados; los puntos de muestreo se realizaron allí donde la concentración de hábitat en disperso era mayor. El muestreo fue intensivo para todos los municipios de las provincias de Almería y la mayoría de Huelva y Jaén (esto es, localizando el número máximo de alojamientos), para el resto de provincias buscamos los alojamientos más próximos a las cuatro esquinas del cuadrado (kilómetro cuadrado). De manera que al final encuestamos a 948 africanos y fueron registradas el mismo número de viviendas.

El cuestionario nunca se realizó en un ámbito que no fuera el residencial, es decir, todas las encuestas se llevaron a cabo en los respectivos alojamientos - y entornos. Alrededor del 75\% de las ocasiones fuimos observadores directos de la vivienda, en el

11 En la provincia de Almería se hizo trabajo de campo en Almería (capital), Adra, Roquetas de Mar, El Ejido, La Mojonera, Vícar, Níjar, Cuevas del Almanzora y Pulpí; en la provincia de Granada: Granada (capital), Zafarraya, Alhama de Granada y Albuñol; en Málaga: Málaga (capital), Marbella, Estepona y Torremolinos; en Sevilla: Sevilla (capital) y Dos Hermanas; en la provincia de Córdoba: Córdoba (capital), Priego de Córdoba, Lucena, Cabra, Puente Genil, Baena y Palma del Río; en la provincia de Jaén: Jaén (capital), Úbeda, Martos, Torre del Campo, Baeza, Villanueva del Arzobispo, Villacarrillo, Linares; en Huelva: Huelva (capital), Almonte, Villablanca, Ayamonte, Lepe, Rociana del Condado, Cartaya, Bonares; y en la provincia de Cádiz: Cádiz (capital) y Algeciras. 
resto de los casos, la información fue obtenida en la puerta del alojamiento. El trabajo de campo en las provincias de Jaén y Córdoba se ejecutó durante la recogida de la aceituna y en Huelva en el periodo de cosecha de la fresa.

En lo concerniente al cálculo de la satisfacción residencial hemos construido un índice tomando como referencia los atributos del barrio (entorno), vivienda y relaciones con los vecinos (Canter y Ress, 1982). Para ello, hemos utilizado; primero, siguiendo el modelo de Amérigo (1990) o Rojo y otros (2001), escalas directas que responden a un único ítem centrado en tres ámbitos de análisis: el entorno más cercano, la propia vivienda y los vecinos, expresadas en cuatro niveles: nada satisfecho, poco satisfecho, bastante satisfecho y muy satisfecho con su barrio, casa y vecinos; y segundo, otra escala de características indirectas ${ }^{12}$ formada por tres ítems: $1 /$ ¿Definiría su barrio como un lugar muy, bastante, algo o nada bueno para vivir?; 2/ Si pudiera hacer cambios en su casa ¿haría muchos, bastantes, algunos o ninguno?; 3/ Si se cambiase de barrio ¿le gustaría encontrarse a todos, casi todos, algunos o ningún vecino actual? También, se preguntó sobre la posibilidad de movilidad residencial atendiendo a su grado de satisfacción.

De manera que aquellos individuos que respondieron a las escalas directas nada o poco satisfecho se dio el valor 0 , al resto 1; y en las escalas indirectas el valor 0 se adjudica a los que contestaron nada bueno en el primer ítem, muchas y/o bastantes en el segundo y ninguno en el tercer ítem. Por último, se asignó un 0 a aquellos que mostraban interés en cambiarse de barrio. En consecuencia, el índice de satisfacción así elaborado oscila entre 0 (nada satisfecho) y 14 (totalmente satisfecho).

Evidentemente, en el cuestionario también se recoge información de predictores concretos del ambiente residencial referidos a equipamientos e infraestructuras básicas del barrio y vivienda, y sobre las relaciones interétnicas (Aragonés y Corraliza, 1992). Así como cuestiones referidas a las propias características de la persona: procedencia, sexo, edad, estado civil, años de estancia en el barrio, amigos en el barrio, familiares en el barrio, ingresos, etc.

Junto al análisis cuantitativo, el trabajo de campo se completó con otro de características cualitativas. Principalmente a través de una observación no participante de los entornos, cuyo resultado se recoge en el apartado de ambiente real y observable.

\section{RESULTADOS}

Cuando tomamos al entorno como objeto de estudio debemos partir del hecho de que éste no es un espacio cerrado y estático, sino un organismo dinámico en continuo cambio, resultado de un proceso evolutivo. Así, las transformaciones que un entorno residencial sufre desde su nacimiento pueden tener dos orígenes. Por un lado, las decisiones individuales y los cambios o transformaciones de la vivienda en base a percepciones subjetivas - de satisfacción - y, por otro, las decisiones institucionales o colectivas de expansión residencial, derivadas de momentos sociohistóricos concretos que se explican

12 Otras investigaciones optan por la elaboración de escalas compuestas por diversos ítems (Vreugenhil y Rugby, 1987). 
por el crecimiento demográfico - inmigración y natalidad - o nuevas coyunturas económicas. Por ello, en este apartado se abordan dos aspectos: primero, el ambiente residencial real, elaborado a partir de investigaciones precedentes y, sobre todo, de observaciones directas en el campo de estudio y, segundo, el subjetivo, a través del establecimiento de dimensiones y predictores de satisfacción residencial que resultan de la posición que los entrevistados tienen en la escala, el cuál será completado, también, con nuestras observaciones.

\section{Ambiente real y observable}

Como se describen en otro lugares (Checa y Arjona, 2005; Checa et al., 2009) los africanos en Andalucía se insertan en dos modelos de hábitat diferentes. Por un lado están los diseminados que son residencias ubicadas fuera de los núcleos urbanos - ciudad dispersa -, en suelo no urbanizable. Construcción residencial que ha sido una constante en toda Andalucía, especialmente en las agrociudades (López, 2002).

Dichos hábitats se encuentran mayoritariamente, como ocurre en las campiñas de Jaén o Córdoba, en emplazamientos escarpados o de relieves enhiestos y casi inaccesibles. $\mathrm{Y}$ en otros casos los cortijos se ubican en zonas llanas, caso de las provincias de Huelva o Almería.

Ahora bien, este tipo de asentamiento tiene manifestaciones diferentes, así por ejemplo, con la observación directa encontramos tanto a los tradicionales cortijos $^{13}$, esto es, viviendas que fueron construidas para dar cobijo a los agricultores y braceros, junto a las explotaciones agrícolas, como a otros tipos de estancias que son utilizadas por los africanos como vivienda, nos estamos refiriendo a los almacenes de productos y aperos del campo, chabolas, autoconstrucciones, naves, lonjas, coches abandonados, etc.

De cualquier modo, y en todos los casos, estamos ante entornos con viviendas aisladas unas de otras, conectadas, en la mayoría de las ocasiones, por caminos o veredas sin asfaltar, iluminar o señalizar, pensados para ser transitados principalmente por animales de carga, tractores, camiones o coches todo-terreno. No obstante, la morfología de esos entornos dista de unos lugares a otros. Si los diseminados en Almería y Huelva se esconden en el laberinto de caminos que dejan las explotaciones minifundistas, en la campiña de Jaén, Córdoba o Sevilla los cortijos se incrustan en las montañas y llanuras que se dedican a la agricultura extensiva - cereal y olivo, principalmente.

Por su lado, los africanos que residen en entornos urbanos, se asientan tanto en los barrios periféricos, construidos inicialmente para la población asalariada y más pobre,

13 No todos los cortijos tienen el mismo origen ni morfología. Si en las campiñas, cordobesa, sevillana y jiennense es un tipo de hábitat que la podemos encontrar siglos atrás, en Almería o Huelva su construcción es mucho más reciente - último tercio del siglo XX -, vinculado, sobre todo, al desarrollo de la agricultura intensiva. De igual modo, la forma de éstos varía ampliamente. Entre los primeros podemos encontrar cortijos que no sólo están configurados por la vivienda del agricultor sino también por la de algún asalariado, zonas de aperos y animales, todo ello en varias dependencias, a otros en los que viven sólo los agricultores y animales en una sola dependencia. En ambos casos las viviendas estaban formadas por varias plantas. Sin embargo, los existentes en Almería o Huelva son más pequeños que los anteriores, muchos de ellos de planta única. 
e incluso en barriadas o entidades locales dependientes separadas del núcleo principal, como ocurre en la provincia de Almería, como en el centro de esas ciudades o pueblos, ocupando los edificios más antiguos y deteriorados. De manera que se insertan en los lugares más denigrados de las ciudades, puesto que a las deficiencias de los propios alojamientos se le une el deterioro y abandono del entorno, especialmente en el caso de los barrios periféricos.

Evidentemente, esta descripción está expuesta al ojo de cualquier observador, pero debe ser completada por la propia definición que de ella hacen los habitantes de esos lugares. No sólo por ser los afectados sino también para tener elementos de trabajo para una posible intervención. De ahí que a partir de ahora abordemos el fenómeno teniendo en cuenta la mirada de los africanos.

\section{Ambiente residencial subjetivo}

En el análisis de este punto queremos determinar, por un lado, los índices de calidad ambiental percibida (por ejemplo, Carp y Carp, 1982; Amérigo, 1990; Aragonés y Corraliza, 1992; Rojo et al., 2001; Adriaanse, 2007) y, por otro, la satisfacción residencial que muestran los africanos en base a esos índices y otras variables.

Se trata, pues, en primer lugar, de conocer las dimensiones que resultan de la evaluación del ambiente residencial por parte de los africanos. Para ello, se realizó un análisis de componentes principales con rotación varimax entre todos los ítems del cuestionario (25) que hacían referencia a esta cuestión ${ }^{14}$. En segundo lugar, medir la satisfacción residencial a partir de dos tipos de escalas, una directa y otra indirecta, y del índice de satisfacción, tal y como hace la literatura precedente.

Empezando por la evaluación que hacen los africanos del ambiente residencial, como muestra la tabla 1 , los factores - dimensiones - resultantes son cuatro ${ }^{15}$, que explican el $66,69 \%$ de la varianza.

El primero queda saturado positivamente por las siguientes variables ${ }^{16}$ : entorno aislado, zona muy deteriorada, iluminación deficiente, calles sucias y transporte público y privado deficiente, las cuales explican el 28,31\% de la varianza. Evidentemente, por las características de estas variables y el signo de la correlación le podemos llamar degradación zonal.

Dicho de otro modo, y constatando la somera descripción previa, los africanos definen sus entornos como deteriorados y aislados, independientemente si lo hacen en diseminados o en núcleos urbanos. Además, las calles - o caminos - como hemos podido comprobar casi siempre están sin limpiar, especialmente los que conectan los diferentes cortijos, ya que no existe un servicio público de limpieza, ni mucho menos iluminación. A esto se debe añadir la inexistencia de líneas de autobús, en ningún caso nos han comentado

14 Del análisis se excluyeron los sujetos que respondían la alternativa ns/nc.

15 Se han eliminado del análisis - y la tabla - los valores que saturan igual o menor a 0,300.

16 Los ítems han sido renombrados y se presentan en forma de variables tanto en el texto como en la tabla para hacerlos más operativos en su presentación y análisis. 
que exista tal servicio para unir estos hábitats con las ciudades o agrociudades más cercanas; de ahí la dificultad que ello acarrea para el desarrollo vital de los individuos trabajo, educación, ocio, etc. Situación que se convierte en dramática, cuando, como nos han comentado los inmigrados, por los parajes han ocurrido accidentes de atropellos de coches a inmigrados por la falta de luz. O la proliferación de ratas por la falta de recogida de basura.

También, en los barrios periféricos de los núcleos urbanos, en el mejor de los casos, llegan dos líneas de autobús, con una frecuencia superior a la media hora. De ahí, que los africanos utilicen, como hemos podido comprobar, la bicicleta o a pie la mayoría de sus desplazamientos. Realidad que se vuelve especialmente penosa cuando éstos realizan la compra semanal los sábados. De manera que bien contratan un taxi entre varios, o se llevan los carros de los supermercados y luego los devuelven vacíos, o en el peor de las situaciones llevan las bolsas de comida andando.

El segundo factor se presenta saturado positivamente por hacinamiento y baja calidad de construcción; negativamente por casa adecuada, casa que ofrece privacidad y vivienda confortable. Atendiendo a estas razones el factor podría denominarse características residenciales.

Por tanto, encontramos que los vecinos detallan graves deficiencias residenciales, tanto en lo que hace referencia a las calidades de la construcción y estructura física de la propia vivienda, como en la escasa privacidad que resulta de dicha arquitectura. Si bien es cierto que en los cortijos los africanos gozan de gran intimidad e invisibilidad, pero se convierten en inadecuados por las distancias entre unos y otros. Hasta el punto que para ver o visitar a algún compatriota los africanos tienen que caminar, en muchos casos, más de tres o cuatro kilómetros por caminos sin asfaltar e iluminar.

Además, con el trabajo de campo hemos podido comprobar que los cortijos, en la mayoría de los casos, presentan graves deficiencias de construcción, bien por su antigüedad, algunos tienen su origen a principios del siglo pasado, bien por la ausencia de reformas, puesto que éstos antes de la llegada de los africanos valían como lugar de los aperos de labranza.

En los centros urbanos, por su lado, estos inmigrantes, habitan barrios periféricos cuyas edificios están muy próximos a otros, dificultando enormemente la intimidad, a lo que se puede sumar que estos edificios tienen muchas habitaciones que se orientan hacia ojos de patios internos en los que las ventanas de unos pisos a otros sólo están separados por unos escasos cuatro metros.

El tercer factor está saturado negativamente por dotación suficiente de parques, servicios públicos y servicios privados. Al índice lo hemos denominado equipamiento del entorno. Esta dimensión, pues, expone con exactitud los parajes y entornos donde se insertan los inmigrantes africanos, puesto que como venimos describiendo, en los diseminados se carece de cualquier infraestructura o equipamiento social y en los barrios urbanos éstos son casi inexistentes o en mal estado. Más concretamente, durante el trabajo no se ha encontrado ningún centro educativo, recreativo, sanitario o de otras características junto a estos entornos. 
Sólo en las zonas urbanas están proliferando, paralelamente al asentamiento de los africanos, un paisaje de comercio regentado por ellos mismos (Arjona, 2006) que abastecen a los compatriotas tanto de productos típicamente étnicos, como del mercado abierto.

El último factor aparece saturado positivamente por buena convivencia, relaciones cordiales entre africanos y autóctonos y solidaridad vecinal. De manera que el nombre del factor es de relaciones de vecindad. Los africanos que ahondaron más en esta temática comentaron que ellos prefieren vivir, mayoritariamente, en barrios ocupados por españoles, puesto que en muy pocos casos han tenido problemas de convivencia. Por tanto, este indicador muestra que las relaciones de vecindad es el único aspecto en el cual los elementos toman un cariz positivo, esto es, los africanos además de establecer buenas relaciones entre ellos, son cordiales con los autóctonos vecinos. Ahora bien, eso no significa, como muestran los propios acontecimientos que no existan ciertos problemas de convivencia, que deben ser tenidos en cuenta a la hora de planificar una intervención urbanística. Puesto que si la opinión de los africanos es positiva, no lo es tanto la de los autóctonos (Rinken y Pérez, 2007; Pérez y Desrues, 2005; Navas y otros, 2004; Herranz, 2008).

Así, por ejemplo, en ciertos barrios donde existe copresencia entre autóctonos y africanos se han sucedido varios acontecimientos violentos. Sin ir más lejos en el barrio de Roquetas de Mar (Almería) de las Doscientas Viviendas un africano murió a manos de un nacional, teniendo como respuesta el ataque de los africanos a los familiares del supuesto asesino. O también en el barrio almeriense de El Puche, los conflictos entre gitanos y africanos, más concretamente, marroquíes es muy frecuente.

Tabla 1: Dimensiones de ambiente residencial

\begin{tabular}{|c|c|c|c|c|}
\hline VARIABLES & Factor 1 & Factor 2 & Factor 3 & Factor 4 \\
\hline Entorno aislado & 0,920 & & & \\
\hline Zona muy deteriorada & 0,835 & & & \\
\hline Iluminación deficiente & 0,627 & & & \\
\hline Calles sucias & 0,632 & & & \\
\hline Transporte público y privado deficiente & 0,588 & & & \\
\hline Hacinamiento & & 0,843 & & \\
\hline Baja calidad de construcción & & 0,789 & & \\
\hline Casa adecuada & & $-0,733$ & & \\
\hline Casa con privacidad & & $-0,433$ & & \\
\hline Vivienda confortable & & $-0,338$ & & \\
\hline Dotación suficiente de parques & & & $-0,931$ & \\
\hline Dotación suficiente de servicios públicos & & & $-0,843$ & \\
\hline Dotación suficiente de tiendas y servicios privados & & & $-0,331$ & \\
\hline Convivencia buena & & & & 0,867 \\
\hline Solidaridad vecinal & & & & 0,437 \\
\hline Relaciones entre inmigrantes y españoles cordial & & & & 0,322 \\
\hline Varianza Explicada & 28,31 & 21,20 & 11,36 & 5,82 \\
\hline $\mathrm{N}=948$ & & & & \\
\hline
\end{tabular}

Fuente: Vivienda, discriminación y segregación espacial de los inmigrados en Andalucía (2008); elaboración propia 
Tanto la varianza explicada, como las variables que saturan a los factores, aunque con combinaciones diferentes, se manifiestan de manera parecida otros estudios empíricos españoles referidos a ámbitos deteriorados. Así, por ejemplo, Amérigo (1990) aísla nueve factores, donde sobresalen, además de la infraestructura básica de la vivienda, similar a nuestro caso, otros como vigilancia e instalaciones deportivas, zonas verdes o infraestructura comercial, los cuales no aparecen de manera independiente para los africanos en Andalucía. Claro está, puesto que los entornos presentan grandes diferencias, si el estudio de Amérigo se centra exclusivamente en lugares urbanos, el nuestro, además, tiene en cuenta parajes situados, en ciertos casos, a muchos kilómetros de cualquier núcleo urbano.

Aragonés y Corraliza (1992), por su lado, aíslan cinco factores. Aunque para este caso el segundo indicador en importancia es el de seguridad ciudadana y el tercero relaciones con los vecinos. Indicadores que cobran la misma importancia en nuestro estudio, puesto que lo más visible y remarcable de los africanos en Andalucía es su segregación en entornos muy degradados.

Por tanto, éstos y otros resultados registrados comprueban, a través de sus similitudes, que tanto la estructura física de la vivienda, como el entorno y las relaciones vecinales son elementos que deben ser tenidos en cuenta a la hora de estudiar la satisfacción residencial. Por supuesto, las diferencias también deben servir como herramienta para seguir trabajando en la mejora de los instrumentos de predicción de la satisfacción residencial.

Una vez determinada la percepción subjetiva del ambiente residencial (entorno, alojamiento y vecindario) de los africanos, nos preocupamos por conocer el grado de satisfacción residencial que presenta este colectivo. Como muestra la tabla 2, haciendo referencia a una escala directa sobre la satisfacción, se puede comprobar que ésta es muy baja. Más concretamente, el $5 \%$ está bastante satisfecho con la zona y/o entorno donde vive y el 1\% muy satisfecho, frente al 29\% que muestra una total insatisfacción y el $65 \%$ poco satisfecho. En cuanto a su casa los valores mejoran sustancialmente con respecto a los datos anteriores ya que el $17 \%$ y $14 \%$ están bastante o muy satisfechos. Sin embargo, el $16 \%$ de los africanos están nada satisfechos con su casa y el $53 \%$ poco satisfechos. Cifras que dejan al descubierto que existe alta coincidencia entre la realidad objetiva barrio en proceso de exclusión y casas con graves deficiencias -, que describimos en el anterior apartado, y la subjetiva (percibida), puesto que el grado de insatisfacción es alto.

Por el contrario, tal y como se mostró en el análisis de los componentes principales, la satisfacción con los vecinos (concepto vacío de contenido para una parte de los africanos que habitan en diseminados) es más alto que el ambiente residencial físico. De modo que el $43 \%$ está bastante satisfecho y el 19\% muy satisfecho; frente al $11 \%$ que declara nula satisfacción y el $27 \%$ poco satisfecho. Por último, y de manera general, la satisfacción con la vida - en general - no es muy acentuada, si bien el $10 \%$ y $27 \%$ están muy y bastante satisfechos, el $12 \%$ no lo está nada y el $51 \%$ poco satisfecho.

En efecto, estos datos evidencian las dificultades y penurias que pasan los africanos en su duelo migratorio, que va desde las condiciones que le invitan a la migración, pasando por los familiares, amigos y recuerdos que dejan hasta las dificultades 
de acomodación que encuentran en la sociedad de llegada, tanto en su inserción laboral, como jurídica, residencial, etc. De ahí que las condiciones residenciales - entorno y casa parece influir con su satisfacción con la vida. Dicho de otro modo, todas estas situaciones hemos podido comprobar que está generando en los africanos diversos y múltiples duelos: soledad, incomprensión, supervivencia, miedo, indefensión, etc., que terminan con serios o graves problemas psicológicos: falta de apetito, insomnio, depresión, etc.

Tabla 2: Satisfacción residencial de los africanos en Andalucía (\%) (escala directa)

\begin{tabular}{|l|c|c|c|c|}
\hline & Nada satisfecho & Poco satisfecho & Bastante satisfecho & Muy satisfecho \\
\hline Con su zona & 29 & 65 & 5 & 1 \\
\hline Con su casa & 16 & 53 & 17 & 14 \\
\hline Con sus vecinos & 11 & 27 & 43 & 19 \\
\hline Con la vida & 12 & 51 & 27 & 10 \\
\hline \multicolumn{1}{|r|}{$\mathbf{N}=\mathbf{9 4 8}$} & \multicolumn{4}{|l}{}
\end{tabular}

Fuente: Vivienda, discriminación y segregación espacial de los inmigrados en Andalucía (2008); elaboración propia

Por su lado, los resultados de la escala indirecta no mejoran los mostrados anteriormente. De manera que sólo el 12\% de los africanos declaran al barrio y/o entorno como bastante o muy bueno para vivir. También cuando se les pregunta sobre la pertinencia de hacer reformas en su residencia sólo el 4\% afirmaba que no necesitaba ninguna reforma, el $46 \%$ valora que serían necesarias bastantes y el 15\% muchas. Cifras que, en cualquier caso, denotan el estado que presentan las residencias. Por último, cuando se pregunta por la composición poblacional del barrio al que se cambiarían si pudieran, el 17\% sostiene que optaría por uno donde estuvieran todos o casi todos los vecinos actuales, el $18 \%$ ninguno y el $65 \%$ algunos. De manera que no se cumple de manera exacta la idea de que cuanto más arraigo existe menor deseo de movilidad y mayor satisfacción. Por tanto, las intenciones de los africanos dejan en evidencia la situación real que padecen, tanto en sus elementos físicos y medioambientales como sociales y poblacionales.

Tabla 3: Satisfacción residencial de los africanos en Andalucía (\%) (escala indirecta)

\begin{tabular}{|l|c|c|c|c|}
\hline \multirow{2}{*}{ Barrio/entorno para vivir } & Nada bueno & Algo bueno & Bastante bueno & Muy bueno \\
\cline { 2 - 5 } & 45 & 43 & 8 & 4 \\
\hline \multirow{2}{*}{ Reformas en la casa } & Ninguna & Algunas & Bastantes & Muchas \\
\cline { 2 - 5 } & 4 & 35 & 46 & 15 \\
\hline $\begin{array}{l}\text { Composición poblacional del } \\
\text { barrio nuevo }\end{array}$ & Ninguno & Algunos & Casi todos & Todos \\
\cline { 2 - 5 } & 18 & 65 & 10 & 7 \\
\hline \multicolumn{2}{|r|}{$\mathbf{N}=\mathbf{9 4 8}$} & \multicolumn{2}{|c}{} \\
\cline { 2 - 5 }
\end{tabular}

Fuente: Vivienda, discriminación y segregación espacial de los inmigrados en Andalucía (2008); elaboración propia 
En efecto, los entornos residenciales - diseminado y barrios periféricos - donde se insertan, principalmente, los africanos en Andalucía son lugares claramente de transición; esto es, lugares que han sufrido el proceso de invasión-sucesión y filtrado del que hablaban los autores clásicos de la Escuela de Chicago. Con el agravante que cuando llegan ya son entornos muy degradados y por la reducida movilidad residencial que están experimentando parecen, también, definitivos. Dicho de otro modo, los africanos expresan que quieren cambiar de viviendas o entornos a otros, con mejores condiciones, pero que encuentran dos frenos insalvables: uno, la falta de capacidad de endeudamiento, puesto que el mercado residencial está guiado por precios muy elevados, al cual sólo se puede acceder a través del hacinamiento o el desempeño de trabajos propios del mercado primario. Y, dos, por la discriminación, debido a que ciertos autóctonos no quieren vivir junto a esta población.

En suma, todas estas cifras de insatisfacción quedan refrendadas con los resultados obtenidos con el índice de satisfacción residencial. Más concretamente, el 7\% de los africanos se muestran nada satisfechos, incluso el $76 \%$ se sitúan en los siete valores más bajos de la escala, hasta el punto que sólo el 1\% de los africanos está muy satisfecho residencialmente. Cifras que se traducen en que el $85 \%$ tiene entre sus planes cambiarse de vivienda, en el momento que pueda.

Tabla 4: Índice de satisfacción residencial de los africanos en Andalucía (\%)

\begin{tabular}{|c|c|c|c|c|c|c|c|c|c|c|c|c|c|c|c|}
\hline & $\begin{array}{c}0 \\
\text { nada } \\
\text { satis- } \\
\text { fecho }\end{array}$ & 1 & 2 & 3 & 4 & 5 & 6 & 7 & 8 & 9 & 10 & 11 & 12 & 13 & $\begin{array}{c}14 \\
\text { muy } \\
\text { satis- } \\
\text { fecho }\end{array}$ \\
\hline Africanos & 7 & 6 & 6 & 9 & 13 & 16 & 12 & 7 & 5 & 5 & 4 & 4 & 3 & 2 & 1 \\
\hline $\mathbf{N = 9 4 8}$ &
\end{tabular}

Fuente: Vivienda, discriminación y segregación espacial de los inmigrados en Andalucía (2008); elaboración propia

Llegados a este punto cabe preguntarse, y como último paso de esta investigación, qué variables y grado de predicción actúan en dicha insatisfacción mayoritaria. Para ello, vamos a tener en cuenta tanto las variables sociodemográficas, como las definidoras de las dimensiones ambientales.

Como se desprende de los datos de la tabla 5, en el modelo uno, en el que sólo se insertan las variables sociodemográficas, las que mejor predicen la satisfacción residencial son, por este orden, amigos y familiares en el barrio/entorno, estancia en el barrio e ingresos con signo positivo; esto es, cuantos más familiares, amigos, años se lleva en el barrio y mejores ingresos mayor es la satisfacción. Por el contrario, el signo negativo aparece con desempleado, de manera que la satisfacción crece con mejores tasas de empleo. Como vemos, la edad, el sexo o el estado civil son peores predictores de la satisfacción, sólo significar que los hombres, los mayores y solteros muestran mayores dosis de satisfacción. De manera que los africanos solteros prestan menor atención a las características de sus 
viviendas - y alrededores - que las mujeres, sobre todo, porque las africanas son las que pasan más tiempo en sus viviendas y entornos. A pesar, incluso, de que los hombres viven más en diseminados que las mujeres.

Por último, el modelo uno, nos informa que, en Andalucía, la satisfacción residencial no queda definida significativamente por el origen, puesto que $\mathrm{P}$ es superior a 0.05. Si bien es cierto que omitiendo dicha regla, los subsaharianos presentan, una mayor satisfacción residencial que los magrebíes.

En el segundo modelo, añadimos el índice de degradación zonal. Como se desprende de la tabla 5, casi todas las variables - excepto calles sucias - son mejores predictoras que las sociodemográficas, especialmente zona deteriorada, entorno aislado, tasas de delincuencia, transporte deficiente, iluminación insuficiente. El signo negativo señala que cuanto más se acentúan estas variables más insatisfacción existe.

En el tercer modelo se añade el factor características y equipamientos residenciales, en el que resalta, con signo negativo, la baja calidad de la construcción, escaso aislamiento del frío y hacinamiento, y con signo positivo vivienda confortable y casa con privacidad. Dicho de otro modo, y como era de esperar, residir en una casa con malas calidades, hacinado y con frío aumenta la insatisfacción frente a vivir en una casa que te ofrece privacidad y es confortable. No obstante, solamente las variables baja calidad en construcción y escaso aislamiento del frío, de esta dimensión, mantiene un grado explicativo alto comparado con el que presentan los valores de la dimensión degradación zonal.

El cuarto modelo incluye variables sobre los equipamientos del barrio, todas con signo positivo. En este sentido una dotación suficiente de servicios públicos, parques y tiendas aumenta la satisfacción. Sobre todo, y para este caso, despunta la dotación de servicios públicos, variable que debería ser tenida en cuenta por los gestores políticos.

El último modelo añade el factor relaciones de vecindad y movilidad residencial, en el que sobresalen, especialmente, las relaciones cordiales interétnicas y movilidad residencial. En menor grado convivencia vecinal buena y la solidaridad vecinal. Por eso, cuanto más conflictivas son las relaciones interétnicas y mayor movilidad social existe, disminuye la satisfacción, situación que se invierte con una buena convivencia y solidaridad vecinal. 
Tabla 5: Predictores de la satisfacción residencial

\begin{tabular}{|c|c|c|c|c|c|c|}
\hline & \multirow{2}{*}{ Variables } & \multicolumn{5}{|c|}{ Modelos } \\
\hline & & Modelo 1 & Modelo 2 & Modelo 3 & Modelo 4 & Modelo 5 \\
\hline \multirow{9}{*}{ 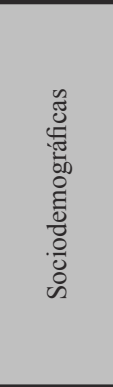 } & Sexo (hombre) & $0.08 * *$ & $0.06 * *$ & $0.09 * *$ & $0.09 * *$ & $0.07 * *$ \\
\hline & Edad & $0.10 * *$ & $0.10 * *$ & $0.10 * *$ & $0.12 * *$ & $0.13 * *$ \\
\hline & Estado civil (casado) & $-0.06 * *$ & $-0.05 * *$ & $-0.05 * *$ & $-0.05 * *$ & $-0.05 * *$ \\
\hline & Desempleado & $-0.21 * *$ & $-0.24 * *$ & $-0.22 *$ & $-0.22 *$ & $-0.21 *$ \\
\hline & Estancia en el barrio (años) & $0.25 * *$ & $0.25 * *$ & $0.25 * *$ & $0.25 *$ & $0.27 *$ \\
\hline & Amigos en el barrio & $0.31 * *$ & $0.33 *$ & $0.33 *$ & $0.34 *$ & $0.35 *$ \\
\hline & Familiares en el barrio & $0.27 * *$ & $0.27 * *$ & $0.26 * *$ & $0.27 * *$ & $0.27 * *$ \\
\hline & Ingresos & $0.19 *$ & $0.19 *$ & $0.20 *$ & $0.20 * *$ & $0.20 * *$ \\
\hline & $\begin{array}{l}\text { Procedencia (subsaha- } \\
\text { rianos) }\end{array}$ & $0.10 * * *$ & $0.10 * * *$ & $0.09 * * *$ & $0.09 * * *$ & $0.09 * * *$ \\
\hline \multirow{6}{*}{ 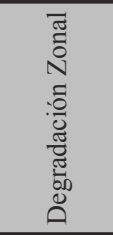 } & Entorno aislado & - & $-0.53 * *$ & $-0.53 * *$ & $-0.53 * *$ & $-0.54 * *$ \\
\hline & Zona muy deteriorada & - & $-0.64 * *$ & $-0.66 * *$ & $-0.65 * *$ & $-0.66 * *$ \\
\hline & Iluminación deficiente & - & $-0.28 * *$ & $-0.28 * *$ & $-0.28 * *$ & $-0.28 * *$ \\
\hline & Calles sucias & - & $-0.10 * *$ & $-0.11 * *$ & $-0.11 * *$ & $-0.11 * *$ \\
\hline & Delincuencia & - & $-0.48 * *$ & $-0.48 * *$ & $-0.48 * *$ & $-0.48 * *$ \\
\hline & Transporte deficiente & - & $-0.37 * *$ & $-0.39 * *$ & $-0.39 * *$ & $-0.39 * *$ \\
\hline \multirow{5}{*}{ 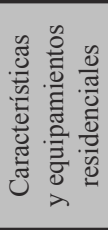 } & Hacinamiento & - & - & $-0.33 *$ & $-0.33 *$ & $-0.33 *$ \\
\hline & $\begin{array}{l}\text { Escaso aislamiento del frío } \\
\text { (inadecuada) }\end{array}$ & - & - & $-0.41 *$ & $-0.41 *$ & $-0.41^{*}$ \\
\hline & Baja calidad construcción & - & - & $-0.64 *$ & $-0.64 *$ & $-0.63^{*}$ \\
\hline & Casa con privacidad & - & - & $0.22 * *$ & $0.22 * *$ & $0.22 * *$ \\
\hline & Vivienda confortable & - & - & $0.29 *$ & $0.29 *$ & $0.29 *$ \\
\hline \multirow{3}{*}{ 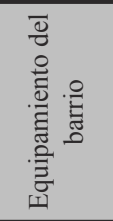 } & $\begin{array}{l}\text { Dotación suficiente de } \\
\text { parques }\end{array}$ & - & - & - & $0.47^{*}$ & $0.47 *$ \\
\hline & $\begin{array}{l}\text { Dotación suficiente de } \\
\text { tiendas }\end{array}$ & - & - & - & $0.40^{*}$ & $0.40 *$ \\
\hline & $\begin{array}{l}\text { Dotación suficiente de } \\
\text { servicios públicos }\end{array}$ & - & - & - & $0.52 *$ & $0.51 *$ \\
\hline \multirow{3}{*}{ 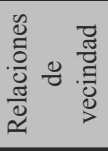 } & Convivencia buena & - & - & - & - & $0.26 * *$ \\
\hline & $\begin{array}{l}\text { Relaciones interétnicas } \\
\text { cordiales }\end{array}$ & - & - & - & - & $0.46 * *$ \\
\hline & Solidaridad vecinal & - & - & - & - & $0.21 *$ \\
\hline \multicolumn{2}{|c|}{ Movilidad residencial } & - & - & - & - & $-0.45^{* *}$ \\
\hline \multicolumn{2}{|c|}{ Coeficiente de Correlación $\mathrm{R}$} & 0.13 & 0.31 & 0.39 & 0.45 & 0.54 \\
\hline
\end{tabular}

* $\mathrm{P}<0.05$

$* * \mathrm{P}<0.01$

$* * * \mathrm{P}<0.09$

$\mathrm{P}$ es la significación de la prueba y sirve para comprobar si el modelo de regresión se ajusta a los datos. Observamos, para nuestro caso, que algunas variables tienen 0.05 y otras 0.01 , pero como trabajamos con una significación por debajo del 0.05 para aceptar el modelo, es decir, existen diferencias entre los residuales y la regresión y, por ello, el modelo se ajusta a los datos. Sólo el lugar de procedencia (magrebíes y subsaharianos) presenta una significación superior, lo que indica que el origen no influye a la hora de predecir la satisfacción.

Fuente: Vivienda, discriminación y segregación espacial de los inmigrados en Andalucía (2008); elaboración propia 
En definitiva, y a la vista de los resultados de todas las variables analizadas, la satisfacción residencial está determinada tanto por los predictores de carácter subjetivo como los objetivos. Más concretamente, y por este orden, ser una zona deteriorada, baja calidad de la construcción, entorno aislado, dotación de servicios públicos, tasas de delincuencia, dotación de parques, relaciones étnicas conflictivas, movilidad residencial, escaso aislamiento del frío y dotación de tiendas son las variables que mejor predicen la satisfacción residencial de los africanos en Andalucía.

El mayor grueso de las variables que predicen la satisfacción en nuestro estudio también las podemos encontrar en otros análisis ${ }^{17}$, tanto nacionales (Amérigo, 1990; Aragonés y Corraliza, 1992, Rojo et al., 2001) como internacionales (Carp y Christensen, 1986; Bruin y Cook, 1997; Rohe y Bassolo, 1997; Carvalho et al., 1997). No obstante, igualmente se muestran algunas diferencias, especialmente las derivadas del sentimiento de apego al lugar, puesto que los años de estancia en el barrio no aparecen como un gran predictor de satisfacción residencial. Está claro, cuanto más apego se tenga al lugar mayor es el grado de satisfacción, pero como hemos comprobado los africanos, por razones obvias, no manifiestan gran apego a su entorno. Es más, el entorno y la vivienda es uno de los elementos fundamentales que empujan a los africanos a tratar de cambiar de vivienda. Éstos entienden que esos lugares de residencia van a ser de transición, donde se pretende estar un tiempo mientras se ahorra y busca otra zona para residir. Esto es, en el proyecto migratorio inicial los inmigrantes africanos sacrifican su calidad de vida en pro de un ahorro que van a invertir en remesas o en una vivienda mejor. Incluso cuando el cambio se produce no conlleva una mejora residencial (Checa, Checa y Arjona, 2009).

Por tanto, sólo la solidaridad vecinal y la buena convivencia actúan de elementos aglutinadores al territorio, pero sin que esto signifique un apego real, puesto que cuando aparezca la oportunidad de cambiar de residencia será aprovechada. Para los africanos el marco ambiental, una vez superado el escollo fenotípico, se convierte en un elemento a sortear para la integración social, puesto que la dirección de la casa se consolida como un indicador del estatus socioeconómico.

\section{CONCLUSIONES}

A partir de los objetivos iniciales planteados se han obtenido varias conclusiones: primera, las características y atributos de los entornos donde se insertan los inmigrados africanos, en las principales ciudades y pueblos andaluces, presentan graves deficiencias en muchos elementos básicos, que convierten a esos hábitats en inadecuados. Más aún, los africanos son uno de los grupos que mayores dosis de segregación presentan en las ciudades y pueblos andaluces.

Segunda, a través de «la mirada de los africanos», los atributos urbanos - de entorno y residenciales - y sociales - relaciones intervecinales - se agrupan en cuatro dimensiones: degradación zonal, características residenciales, equipamiento del entorno y relaciones de vecindad.

17 No obstante, el coeficiente de correlación $\mathrm{R}^{2}$ de nuestro estudio es superior a los obtenidos por gran parte de los estudios citados. 
Tercera, el grado de satisfacción residencial de los africanos en Andalucía es muy bajo. Más concretamente, ningún africano está totalmente satisfecho residencialmente, de manera que los siete valores más bajos de satisfacción de la escala agrupa al 76\% de los africanos.

Cuarta, la satisfacción residencial se explica tanto por indicadores objetivos como subjetivos, aunque resaltan por su importancia: zona deteriorada, baja calidad de la construcción, entorno aislado, dotación de servicios públicos, tasas de delincuencia, dotación de parques, relaciones étnicas conflictivas, movilidad residencial, escaso aislamiento del frío y dotación de tiendas. Hasta el punto, que la diversidad de procedencia agrupada en subsaharianos y magrebíes - no es significativo a la hora de predecir dicha satisfacción.

Última, la movilidad residencial entre los africanos es una opción clara como respuesta a la insatisfacción residencial, que en la mayoría de los casos se ve frenada por sus posibilidades económicas o actitudes de discriminación de la población autóctona.

En definitiva, los resultados muestran que la satisfacción/insatisfacción residencial por es un elemento fundamental a la hora de elaborar políticas residenciales de mixticidad. No obstante, a pesar de que en esta investigación los resultados de la ecuación predicen más de la mitad de la varianza, se debería seguir investigando otras variables que puedan incidir en la satisfacción residencial, con el propósito final de contribuir al diseño de políticas urbanísticas que planteen entornos más adecuados y habitables.

\section{Referencias bibliográficas}

ADRIAANSE Carlinde (2007) Measuring residential satisfaction: a residential environmental satisfaction scale (RESS), Housing Built Environ, 22, pp. 287-304.

AMÉRIGO María (1990) Satisfacción residencial. Una aproximación Psicosocial a los estudios de calidad de vida, Madrid, Universidad Complutense, $344 \mathrm{p}$.

AMÉRIGO María and ARAGONÉS Juan Ignacio (1990) Residential satisfaction in council housing, Journal of Environmental Psychology, 10, pp. 313-325.

AMÉRIGO María and ARAGONÉS Juan Ignacio (1997) A theoretical and methodological approach to de study of residential satisfaction, Journal Environmental Psychology, 17, pp. 47-57.

ARAGONÉS Juan Ignacio y CORRALIZA José (1992) Satisfacción residencial en ámbitos de infravivienda, Psicothema, 4 (2), pp. 329-341.

ARJONA Ángeles (2006) Los colores del escaparate. Emprendedores inmigrados en Almería, Barcelona, Icaria, $239 \mathrm{p}$.

BAKER Emma (2008) Improving outcomes of forced residential relocation: The development of an Australian tenants' spatial decision support system, Urban Studies, 45 (8), pp. 1712-1728.

BALDASSARE Mark (1986) Residential satisfaction and the community question, Sociology and Social Research, 70 (2), pp. 139-142.

BERUBE Alan (2005) Mixed Communities in England. A US perspective on evidence and policy prospects, London, Joseph Rowntree Foundation, 73 p.

BONAIUTO Marino, AIELLO Antonio, PERUGINI Marco, BONNES Mirilia and ERCOLANI Anna Paola (1999) Multidimensional perception of residential environmental quality and neighbourhood attachment in the urban environment, Journal of Environmental Psychology, 19, pp. 331-352. 
BRUIN Marilyn and COOK Christine (1997) Understanding constraints and residential satisfaction among low-income single-parent families, Environment and Behavior, 29 (4), pp. 532-553.

CAMPBELL Angus, CONVERSE Philip and RODGERS Willard (1976) The quality of American life: perceptions, evaluations and satisfactions, New Cork, Russel Sage Foundation, 564 p.

CANTER David and RESS Kevin (1982) A multivariate model of housing satisfaction, International Review of Applied Psychology, 31, pp. 185-208.

CARP Frances and CARP Abraham (1982) A role for technical environmental assessment in perceptions of environmental quality and well-being, Journal of Environmental Psychology, 2, pp. 171-191.

CARP Frances and CHRISTENSEN David (1986) Technical environmental assessment predictors of residential satisfaction. A study of elderly women living alone, Research on Aging, 8, pp. 734-768.

CARVALHO Mayra, GEORGE Varkki and ANTHONY Kathyn (1997) Residential satisfaction in condominios exclusives (gate-guarded neighborhoods) in Brazil, Environment and Behavior, 29 (6), pp. 734-768.

CHAPMAN David and LOMBARD John (2006) Determinants of neighborhood satisfaction in fee-based gated and nongated communities, Urban Affairs Review, 41 (6), pp. 769-799.

CHECA y OLMOS Francisco, CHECA Juan Carlos y ARJONA Ángeles (2009) Residencia e inmigración en Andalucia: viejos problemas, nuevos afectados, Barcelona, Enthinema, 182 p.

CHECA Juan Carlos (2007) Viviendo juntos-aparte. La segregación espacial de los africanos en Almería, Barcelona, Icaria, $212 \mathrm{p}$.

CHECA Juan Carlos y ARJONA Ángeles (2005) El vecino no deseado. Situación residencial de los inmigrantes africanos en Almería (España), Revue Européenne des Migrations Internationales, 21 (3), pp. 179-208.

COLECTIVO IOÉ (2005) Inmigración y vivienda en España, Madrid, Ministerio de Trabajo y Asuntos Sociales, $167 \mathrm{p}$.

CORTÉS Luís (1995) La cuestión residencial. Bases para una sociología del habitar, Madrid, Fundamentos, $345 \mathrm{p}$.

CUTTER Susan (1982) Residential satisfaction and the suburban homeowner, Urban Geography, 3, pp. 315-327.

FRIED Marc and GLEICHER Peggy (1961) Some sources of residential satisfaction in an urban slum, Journal of American Institute of Planners, 27, pp. 305-315.

GALSTER George and HESSER Garry (1981) Residential satisfaction: compositional and contextual correlates, Environment and Behavior, 13, pp. 735-758.

GIFFORD Robert (1987) Environmental psychology. Principles and practice, Massachussets, Allyn \& Bacon, $214 \mathrm{p}$.

HERRANZ Gonzalo (2008) Xenofobia: Un estudio comparativo en barrios y municipios almerienses, Revista Española de Investigaciones Sociológicas, 121, pp. 107-132.

HOLLINGSHEAD August and ROGLER Lloyd (1963) Attitudes toward slums and public housing in Puerto Rico, in Leonard Duhl Ed., The urban condition, New York, Basic Books, pp. 229-245.

KARSTEN Lia, REIJNDORP Arnold en VAN DER ZWAARD Joke (2006) Smaak voor de stad, een studie naar de stedelijke woonvoorkeur van gezinnen, The Hague, Ministerie van VROM.

KEMENY John (1992) Housing and social theory, London, Routledge.

KENNA Padraic (2006) Los derechos a la vivienda y los derechos humanos, Barcelona, Prohabitatge, $321 \mathrm{p}$.

LECKIE See (2000) Legal resources for housing rights, Ginebra, COHRE.

LIAO Peter (2004) Emotion attachment, residential satisfaction and mobility propensity, Journal of Population Studies, 28, pp. 49-79.

LÓPEZ Antonio (2002) Las agrociudades andaluzas, in Eduardo Moyano y Manuel Pérez Eds., La sociedad andaluza, Córdoba, IESA, pp. 285-305. 
MARANS Robert W. and RODGERS Sam (1975) Toward an understanding of community satisfaction, in Amos Hawley and Vincent Rock Eds., Metropolitan America in contemporary perspective, New York, Halstead Press.

MARTÍNEZ Manuel, FELKA Stuart, SIMPSON-HOUSLEY Paul and DE MAN Anthony (1991) Neighbourliness, socializing and residential satisfaction in urban settings: Two studies of German and Spanish immigrants, International Journal of Comparative Sociology, 32 (3), pp. 310-310.

MCCREA Rod, STIMSON Robert and WESTERN John (2005) Testing a moderated model of satisfaction with urban living using data for Brisbane-south east Queensland, Australia, Social Indicators Research, 72 (2), pp. 121-152.

NAVAS María Soledad y PUMARES Pablo et al. (2004) Estrategias y actitudes de aculturación: la perspectiva de los inmigrantes y de los autóctonos en Almería, Sevilla, Junta de Andalucía, $335 \mathrm{p}$.

NEWMAN Sandra y DUNCAN Greg (1979) Residential problems, dissatisfaction, and mobility, Journal of the American Planning Association, 45, pp. 154-166.

$\mathrm{OH}$ John (2003) Social Bonds and the Migration Intentions of Elderly Urban Residents: The Mediating effects in residential satisfaction, Population Research and Policy Review, 22 (2), pp. 127-146.

PARKES Alison, KEARNS Ade and ATKINSON Rowland (2002) The determinants of neighbourhood dissatisfaction, CNR, Paper 1.

PÉREZ YRUELA Manuel y DESRUES Thierry (2005) Opinión de los españoles en materia de racismo y xenofobia, Madrid, Observatorio Español de Racismo y Xenofobia, 149 p.

PINQUART Martin and BURMEDI David (2004) Correlates of residential satisfaction in adulthood and old age: A meta-analysis, Annual Review of Gerontology and Geriatrics, pp. 195-222.

PHILLIPS David, SIU Oi, ANTHONY Yeh and CHENG Kevin (2004) Factors influencing older persons' residential satisfaction in big and densely populated cities in Asia: A case study in Hong Kong, Ageing International, 29 (1), pp. 46-70.

ROE Walter and BASOLO Victoria (1997) Long-term effects of homeownership on the selfperceptions and social interaction of low-income persons, Environment and Behaviour, 29, pp. 793-819.

RINKEN Sebastian y HERRÓN Anais (2004) La situación residencial de la población inmigrante en Andalucía, Revista Internacional de Sociología, 38, pp. 7-31.

ROJO Fermina, FERNÁNDEZ-MAYORALAS Gloria and POZO Enrique (2001) Ageing in place: predictors of residential satisfaction of elderly, Social Indicators Research, 52 (2), pp. 173-208.

RYAN Edward (1963) Personal identity in an urban slum, in Leonard Duhl Ed., The urban condition, New York, Basic, pp. 135-150.

SPEARE Alden (1974) Residential satisfaction as an intervening variable in residential mobility, Demography, 11 (2), pp. 173-188.

TUNSTALL Rebeca and FENTON Alex (2006) In the mix. A review of mixed income, mixed tenure and mixedcommunities, Joseph Rowntree Foundation \& Housing Corporation.

VREUGDENHIL Anthea and RIGBY Kenneth (1987) Assessing generalized community satisfaction, The Journal of Social Psychology, 127 (4), pp. 367-374.

WEIDEMANN Strotmann and ANDERSON Elhendy (1985) A conceptual framework for residential satisfaction, in Irwin Altman y Carol Werner Eds., Home Environments, New York, Plenum Press.

WILLMOT Peter and YOUNG Michael (1960) Family and class in a London suburb, London, Routledge \& Kegan Paul.

YANCEY Clyde (1971) Architecture, interaction and social control: the case of a large-scale public housing project, Environment and Behaviour, 3 (1), pp. 3-21. 